\title{
Semantic Network-based Approach to Studying the Choice of Lexis in Definitions of Technical Terms
}

\author{
Maxim Nikolayevich Latu (Corresponding author) \\ Western European Languages and Cultures Department, Pyatigorsk State University, Russia \\ E-mail: maxim.latu@mail.ru \\ Aleksey Valeryevich Razduev \\ Western European Languages and Cultures Department, Institute of Translation Studies and Multilingualism, Pyatigorsk State University, Russia \\ Received: 02-08-2016 \\ Published: 10-12-2016 \\ Accepted: 20-10-2016 \\ doi:10.7575/aiac.ijalel.v.5n.7p. 153 \\ Advance Access Published: November 2016 \\ URL: http://dx.doi.org/10.7575/aiac.ijalel.v.5n.7p.153
}

\begin{abstract}
This paper is devoted to the study of vocabulary units that are used in definitions of technical terms with the help of semantic network analysis. Since a semantic network represents a model of a definite system of knowledge it is claimed that it may reveal what predetermines the choice of adjacent technical terms as well as words of general vocabulary in definitions of technical terms denoting academic concepts that belong to different categories. The research demonstrates how some prototypic semantic schemes that consist of semantic relations of definite type between the adjacent technical terms of certain categories are reflected in definitions. The examples are mainly drawn from the actively developing terminologies of nanotechnology and space research as well as physics when necessary.
\end{abstract}

Keywords: technical term, category, semantic relation, concept, terminology

\section{Introduction}

As the scientific and technological progress keeps changing the world, new fields of knowledge and new concepts appear very fast and in large numbers. These are expressed by a bulk of new technical terms that form terminologies and need to be properly defined. Such topical scientific fields as nanotechnology and space research contribute largely to the process with some of the technical terms being used beyond the borders of academic discourse and recognizable by an audience of non-specialists. Still, specialist lexis remains the determining factor of a specialist language (Wessels 2010: 200) and allows professionals to communicate on a highly technical level (Staicu 2010: 175). As it is known, a definition of a technical term outlines the scope of the concept as it is opposed to similar and adjacent concepts in the terminology system. In a very general sense definitions tell what words people use under what conditions (Hayakawa 1990: 88). Quite often dictionaries and other specialized sources offer definitions of one and the same concept that do not coincide at least in the vocabulary used, to say nothing of length, correctness and fullness when one definition may complement another.

Semantic networks model the organization of naïve and academic knowledge (Latu 2013), and the sphere of their application today is becoming wider ranging from attempts to develop a semantic web to the construction of terminological ontologies, electronic dictionaries, etc. These may be based on different principles, for example, synsets and hyponymy relations as in the case of WordNet (Fellbaum 1998) or links that demonstrate derivative processes when one term occurs in another forming networks of natural hierarchies (Lande 2014). Technical terms represent academic concepts of a particular scientific field as opposed to the words of general vocabulary. Semantic network of a terminology system reflects the organization of technical terms belonging to different categories linked up by a set of semantic relations (Latu 2016, 116). Applying it to the study of technical term definitions may shed light on what systematically adjacent technical terms appear in definitions to specify some or other technical term and which of them do not as well as the peculiarities of general and specialized vocabulary interaction.

\section{Method}

The research relies on general principles of semantic networks building that are reflected in the works by $\mathrm{S}$. Khabarov, M. Steyvers, J.B. Tenenbaum. At the same time we put emphasis on technical terms each of which refers to a predefined category of mental constructs (scientific concepts). Their referents may be material (of natural origin: Object of natural environment, natural Substance, Locus and man-made: Instrument, Mechanism, man-made Material, manmade Locus/Construction) or non-material (Process, Characteristic, Ideal phenomenon, Situation and Actor). We see these categories as types of vertices of the semantic network. Similarly, we used the types of arcs that are typical and common semantic relations between the technical terms of the same and different categories in a terminology: AKO "a kind of" (the relation between the generic term and its direct hyponyms), ISA - "is a" (the relation expressing concurrence or inclusion into some aggregate that is not the direct generic term), $P O$ - "part of" (the relationship of meronymy, links the referents that are considered to be a part of another referent), At - "attribute" (the relation with 
characteristics and properties), $A g$ - "agent" (the relation linking the process with the doer), $O b j-$ "object" (the relation between the process and the object under influence), $L o c$ - "locus" (the relation pointing at the location), $S r$ - "source" (pointing at the initial position of the referent), Rec - "recipient" (links the referent with the concept expressing the final position), Inst - "instrument" (a relation with the referent that is seen as an intermediary or an instrument to attain some aim), $R$ - "result" (the relation linking the referents seen as the reason and the result). These semantic relations demonstrate the organization of technical terms within a terminology as well as the adjacent concept that they express. In this study we focus mainly on astrophysics, nanotechnology as well as physics that correlate with both of them and their terminologies from which most of the examples are drawn.

Primarily technical terms and their definitions from various specialized sources including dictionaries were collected. As we faced the fact that definitions of one and the same concept could be incomplete, differ by structure and in content in different sources, we selected no less than three definitions of each technical term. The overall selection comprised 370 technical terms and 1,314 definitions. As it was confirmed that in many cases some important information may be omitted in one definition and be expressed in another definition, all of them were analyzed to get a full picture of the whole set of the defining features. Throughout the study it was taken into account that some definitions of technical terms (especially of ad hoc concepts) may be aimed at scholars and specialists only, while those of popular, socially significant or widely known concepts may be aimed at non-specialists. Then the vocabulary used in the definitions was classified according to lexical strata. Technical terms used in the definition were selected and counted. It was consequently analyzed what pieces of knowledge are expressed by technical terms and what by vocabulary of general use, as well as what predetermines the choice. Then, they were systemized according to the essence and nature of the referents they express and, consequently, subdivided into the predefined categories that constitute the set of vertices types.

The next step was the identification of the semantic relations that link the defined technical term with those mentioned in its definitions and the conceptually adjacent technical terms that appear in specialized texts. Based on these constituent elements, the semantic network was built. A semantic relation of a certain type linking up two vertices of certain types (Latu 2016, 115) (such as a Process and a Natural object linked up by "result" semantic relation or a Natural object and a Substance linked up by "part of" relation) we consider a semantic scheme of concept interaction. Then, some prototypic schemes that appear or potentially may appear in definitions and those that do not were considered.

\section{Results and discussion}

What technical terms appear in the definition largely depends on the concept itself. First of all, an important factor is surely the field of knowledge it refers to. All other technical terms of the scientific sphere that are used in the definition, clearly demonstrate the systemacy of concepts. Secondly, the exact technical terms as well as the words of the general vocabulary used in the definition depend on the scope of concept and the specific features that define it as a separate concept as compared to other concepts referring to the same generic term. It is quite expectable that not all the features that have the potential to characterize the referent appear in the definition but only those that are important to define it as a concept of the field from a specialist's point of view. Concepts of a particular field of knowledge do not originate all of a sudden out of the blue, but always are a result of some research and time-consuming analysis that is grounded on the ideas that have been expressed and proved earlier. So the concept under discussion when it is incorporated into the sphere of knowledge is always related to some adjacent concepts expressed by technical terms of the respective field. The fact is again based on the systematic character of the scientific vocabulary. However a concept cannot be equally linked with all the concepts that constitute the given scientific field, but can only with a limited set of adjacent concepts. For example, the technical term cryovolcanism (Process) is adjacent to the technical terms planet (Natural object), natural satellite (Natural object) but has no evident relation with the technical terms luminosity (Characteristic) or elliptic orbit (Locus) or space station (Construction).

Finally, a significant factor that to some extent determines the peculiarities of technical terms use within the scope of a definition is the category of mental constructs to which the interpreted concept refers. Thus, many referents of material nature that constitute the categories Instrument, Mechanism, Natural object, when incorporated into a classification based on hyponymy (the so called "a kind of" arc that links the hyponyms with generic concepts in a semantic network), have generic concepts expressed by other technical terms. For example, a single-walled nanotube and a multiwalled nanotube is a kind of a carbon nanotube which is in turn a kind of nanotube together with other kinds of noncarbon nanotubes. Similarly, nanotubes are a member of a whole cluster of 1-D nanoobjects a kind of nanoobjects, etc. As a rule, the information based on this important taxonomic semantic relation is represented in definition following Aristotle's conception of definition construction. This is the principle when you keep abstracting and go up the abstraction ladder (Hayakawa, 1990, 86).

Technical terms that refer to such categories of mental constructs as Actor, Process or Characteristic do not normally form multileveled hierarchical classifications based on this semantic relation and may not have a generic concept that is expressed by a technical term of the same field of knowledge. Thus, such a generic concept may be represented by a technical term that belongs to another terminology, be expressed by an interdisciplinary technical term or simply a word of general vocabulary. For example, the technical term cosmic radiation does not have the direct generic concept represented by a technical term of the terminology of space research. The underlying form makes clear that it is a form of radiation which is an interdisciplinary technical term originally from physics but also used in medicine, IT, etc. However radiation is not the direct generic concept. The definition reveals that it is electromagnetic or corpuscular 
radiation that has an extraterrestrial source. The technical term electromagnetic radiation is a term of physics and is a hyponym of radiation. The technical term radiation does not have a direct generic concept expressed by a technical term and is defined with the words of general vocabulary such as "process", "act", "flow". Sometimes when the generic concept is reflected in the underlying (inner) form of the defined technical term it is occasionally omitted in its definition. For example, "electromagnetic radiation is the flow of energy at the universal speed of light through free space or through a material medium in the form of the electric and magnetic fields that make up electromagnetic waves such as radio waves, visible light, and gamma rays (Encyclopedia Britannica) where the technical term is directly defined by means of a word of general vocabulary "flow".

The same is true for the technical term thermal conductivity that is a characteristic of many objects but at the same time is a distinctive feature (valuable and significant for scientific knowledge development) of certain objects including nanotubes in nanotechnology sphere. It may be linked to a very general concept expressed by a word combination "physical properties" but some definitions either have no reference to the concept considered to be generic or represent it by means of words of general vocabulary: thermal conductivity - the amount of heat per unit time per unit area that can be conducted through a plate of unit thickness of a given material (Dictionary.com), property of a material to conduct heat (Your Dictionary).

It is worth noting that a defined concept may also appear to be a generic concept for other concepts that are its hyponyms as it has been demonstrated in some of the previous examples. Apart from that it may have hyponyms that refer to more than one classification based on different distinctive features, for example various classifications of stars. The AKO relation with the hyponyms of the defined concept and the technical terms that express these hyponyms are much rarely used in technical terms definitions. For example, cosmic rays are subdivided into primary cosmic rays and secondary cosmic rays, but this information is not mentioned in its definition.

It is obvious that the semantic network of a terminology system may reveal far more semantic relations between various concepts expressed by technical terms than represented in a definition. For example, a concept of the category Characteristic may characterize a number of different referents with no specific technical term for the whole group. For example, thermal conductivity is a feature of nanowires, nanotubes, graphite, polymer-nanotube composites, fluorographane, etc. only within the nanotechnology terminology (Razduev 2015), so in the above definition all these are united under a word of general vocabulary "material". Similarly, there may be many concepts that are considered to be the sources of a process. For example, black holes, neutron stars, pulsars, supernovae, active galactic nuclei, quasars, the Big Bang are considered to be the sources of primary cosmic rays and are related to this technical term by a corresponding arc in the semantic network. All these are united under a word combination "astrophysical source" with a very broad sense: "primary cosmic rays are stable charged particles that have been accelerated to enormous energies by astrophysical sources" (The cosmic connection). Nevertheless some processes may be associated with very specific concepts represented by technical terms that are considered to be the sources, constituent parts, loci, etc. that appear in the definition because they serve as the defining features. Thus, stellar wind is defined as "the radial outflow of ionized gas from a star" (Dictionary.com), linking the process with the concept that is considered to be its source and is expressed by a technical term - star. Similarly, thermonuclear fusion is related to stars by means of "locus" arc because that is the exact place where it occurs in astrophysics with the fact mentioned in its definition.

This brings us to the problem of studying the prototypic schemes of technical terms interaction that belong to different categories. Thus, a technical term of the category Characteristic can be related to the technical terms (if any) that represent the generic concept and hyponyms by means of AKO ("a kind of") relationship. It can also be related to a concept of various categories it characterizes by means of At ("attribute") relation, to concepts (such as Mechanism or Ideal phenomenon to which various units of measurement refer) that are used to measure this Characteristic by means of Inst ("instrument") relation. Schemes vary for different categories of mental constructs and not necessarily all of them are present in definitions. Still they represent the potential relations that a technical term of a given category may set up. These also link it with conceptually adjacent technical terms. For example, the technical term electric tension/voltage characterizes electric current by means of At relation and is connected to the technical terms volt (Ideal phenomenon) and voltmeter (Mechanism) by means of Inst relation. According to the Oxforddictionaries.com, electric tension is "The property by virtue of which an electric current tends to flow; a difference of electric potential" (Oxforddictionaries.com). This definition mentions the technical term electric current the characteristic is associated with, but doesn't include the Inst relation. In Collins Cobuild Dictionary we find "the voltage of an electrical current is its force measured in volts" (Collins Cobuild Dictionary) where this information is included. Both definitions do not involve information about the voltmeter. It is worth noting that the definitions of the technical terms that belong to the Characteristic category as a rule do not include this information. Concepts that belong to other categories may have other semantic relations some of which may be reflected in definitions, for example, a PO relation that links up Natural object and Natural substance (any substance of natural origin), Instrument and Material (any man-made material or substance), etc. Similarly, Processes may have Ag ("agent"), Obj (“object"), R ("result") relations with a number of categories such as Mechanisms, Instruments, Materials, etc. They may occur in places that are a specific Locus or Natural object technical terms, depending on the concept.

So it is quite clear that not all semantic relations and the adjacent concepts appear in definitions. The scope of a definition is limited by the exact goal to contrast and define the differences with the adjacent concepts of the same category. Still the other adjacent concepts that are linked up with the defined concept may well appear in an encyclopedic article or specialized literature. For example, micromorphology is a characteristic which is a set of form 
and structural features of a whole class of nanoobjects that is studied by means of optical microscopy or electron microscopy (all this information is reflected in its definition). Among the nanoobjects are nanoparticles that are also linked to the micromorphology according to the inheritance principle. This fact is reflected in some definitions of micromorphology but does not appear in the definitions of nanoparticles. What technical terms actually do are dimension and nanometer: "between 1 and 100 nanometers in size". This interval is also expressed by a single term nanoscale that does not appear in the definitions but potentially could be used instead of the whole phrase. The technical term nanoparticle is linked up with a variety of processes among which are different activities for their production such as disintegration. This relation however is not reflected in the definitions, for there are many processes related to nanoparticles as well as other products of disintegration and there is no necessity to specify all of them. The mechanism disintegrator is related to both nanoparticle by means of Inst relation since it acts as a means to produce these nanoscale objects and disintegration by means of $\mathrm{Ag}$ relation because it is the agent of the process. Its definitions describe the process of disintegration but do not mention the technical term itself. Nanoparticles or nanostructured materials (Rusnano.com) are mentioned in some definitions when its functions are described.

\section{Conclusion}

The analysis has demonstrated that semantic network is an effective means to study the content of definitions as well as organization of adjacent concepts. The academic knowledge represented in technical term definitions is expressed by the lexis that refers to different strata. Their choice is to some extent predetermined by a number of factors. On the one hand, these are technical terms that predominantly belong to the same terminology (thus clearly illustrating the systematic character of its units) or certain adjacent terminologies when two fields of knowledge have a common area where they overlap. On the other hand, these are words of general vocabulary that express the information that cannot be or have no necessity to be expressed by the technical terms of the terminology. At the same time we have to notice that the ratio between the two may vary depending on several factors. Definitions of the technical terms that express socially significant or popular concepts that travelled beyond the borders of academic discourse and are aimed at nonspecialists may contain no technical terms at all, explaining the idea in simple words of general vocabulary. Definitions aimed at specialists as a rule contain at least one technical term with their number depending upon the fullness of the definition, the number of adjacent concepts that mark the specificity of the defined concept and the category of mental constructs the concept belongs to. Both definitions aimed at specialists and non-specialists may differ in length and in content. What technical terms are used also depends on several factors. It is the specificity of the field of science the concept refers to as well as its volume. A significant influence is exerted by the category of the mental constructs the defined concept refers to and the schemes of interaction based on the set of semantic relations with the adjacent concepts of the system that mark the specificity of the defined concept. A semantic network helps to identify the adjacent concepts and the semantic relations between them. Each category has a specific set of prototypic schemes that link up the defined term with the adjacent terms of other categories. However only those technical terms appear in the definition that are seen as marking the specificity and uniqueness of it among those having similar properties. Human factor also has its influence because dictionaries as well as other specialized sources offer definitions that may differ from one another in fullness and correctness. Identifying the schemes that are typically represented in definitions of technical terms that belong to different categories and providing explanation why this happens have been beyond the scope of this research. However it represents a fundamental objective for further studies because it may shed light on what structural fragments of academic knowledge organization are expressed when technical terms of different categories are defined.

\section{Acknowledgements}

The authors express their gratitude to the Ministry of education and science of the Russian Federation (project No. 14.Z56.16.4913-MK "The integrated cognitive study and development of prototypic technical term definition model based on frame and semantic network analysis") and Russian Scientific Fund (project No. 15-18-10006 "The research of anthropocentric nature of language in the cognitive context") that financially supported the study.

\section{References}

Collins Cobuild Advanced Learner's English Dictionary. (2008). New digital edition. HarperCollins Publishers.

Dictionary.com. Retrieved June 15, 2016 from http://www.dictionary.com.

Encyclopedia Britannica. Retrieved June 15, 2016 from https://global.britannica.com/science/electromagnetic-radiation Fellbaum, C. (1998). WordNet: An electronic lexical database. Cambridge, MA: MIT Press, pp. 423.

Hayakawa, S. I. (1990). Language in thought and action. San Diego: Harcourt, pp. 196.

Khabarov, S. P. (n. d.). Semantic networks. Retrieved June 15, 2016 from http://www.habarov.spb.ru/bz/bz05.htm

Lande, D. V. (2014). Building of networks of natural hierarchies of terms based on analysis of texts corpora. E-preprint arXiv 1405.6068 .

Latu, M. N. (2013). Mirroring of cognitive bonds and its reflection in the language. Middle East Journal of Scientific Research, 16 (12), 1681-1685. 
Latu, M. N. (2016). Towards the possibility of defining prototype schemes of technical terms relations in semantic networks. The Strategies of Modern Science Development: Proceedings of the IX International scientific-practical conference. North Charleston, SC, USA, 12-13 April 2016. North Charleston: CreateSpace. 115-118.

Oxforddictionaries.com Language matters. Retrieved June $15, \quad 2016 \quad$ from http://www.oxforddictionaries.com/definition/english/electric-tension?q=electric+tension

Razduev, A. V., Latu, M. N., \& Mironenko, Y. V. (2015). Evolution and present state of the English nanotechnology technical terms. European Journal of Science and Theology, 11 (3), 79-88.

Rusnano thesaurus. Glossary of nano technology and related terms. Retrieved June 15, 2016 from http://eng.thesaurus.rusnano.com/search/?q=Nanoparticles+or+nanostructured+materials

Staicu, S. N., (2010). Technical vocabulary of medicine. Annals of the University of Craiova, 11 (1), 165-176.

Steyvers, Mark, Tenenbaum, \& Joshua.B. (2005). The large-scale structure of semantic networks: statistical analysis and a model of semantic growth. Cognitive Science, 29, 41-78.

The cosmic connection. Primary Cosmic Rays. Retrieved June 15, 2016 from http://cosmic.lbl.gov/SKliewer/Cosmic_Rays/Primary.htm

Wessels, D. (2010). Issues of lexicology in language for special purposes. Annals of the University of Craiova, 11 (1), 198-213.

Your Dictionary. Examples of Physical Properties. Retrieved June 15, 2016 from http://examples.yourdictionary.com/examples-of-physical-properties.html\#LVxjhLZQt1WfeR0t.99 